\title{
WIND TURBINE COMPOSITE BLADE: FRACTURE MECHANICS ASSESSMENT
}

\author{
WALEED AHMED* \\ Mechanical Engineering Department, United Arab Emirates University, \\ Al Ain, Abu Dhabi, UAE
}

[Received: 13 August 2019. Accepted: 30 April 2020]

doi: 10.7546/JTAM.50.20.03.03

\begin{abstract}
The study aims to investigate a wind turbine composite blade subjected to wind loading forces. Linear elastic fracture mechanics principle was used to evaluate the blade with a deteriorated adhesive layer through the estimation stress intensity factor of an edge crack at the adhesive layer. The opening and sliding mode of SIF were predicted and discussed. Two dimensional, plane strain FE modeling was adopted in the study. Interfacial stresses along the bonding line of the composite blade were estimated as well. A parametric study was conducted to assess the influence of the adhesive, mechanical properties on the levels, and the distribution of the stresses along the interfacial bonding line of the composite blade for both intact and fractured blade.
\end{abstract}

KEY WORDS: Wind turbine, composite blade, fracture mechanics, FEA.

\section{INTRODUCTION}

During the operation of wind turbines, blades are subjected to complicated loading conditions caused by the nature of wind. Wind turbine technology can offer an affordable as well as an alternate renewal energy source due to its capability of producing greater amounts of electrical energy with a quit zero greenhouse effects in comparison to other energy generating technologies including solar cell, biofuel, hydrogen, tidal wave, biodiesel, and biomass technologies [1]. Wind turbine simply transforms the kinetic energy of the wind into electrical energy, where output power delivered to a transformer, which converts the electricity from the generator to the appropriate voltage for the power collection system [2]. There are many advantages of renewable energy sources because they can offer clean, uninterrupted, environmental impact-free electrical energy at a reasonable cost. Studies showed that wind and solar sources offer the cleanest and most cost-effective renewable energy, pollution-free electricity generation, fast installation, and commissioning capability, low operation and maintenance cost, and using free and renewable energies [3]. In general, energy crisis and global warming have led to a higher demand for renewable energy [4], so

\footnotetext{
${ }^{*}$ Corresponding author e-mail: w.ahmed@uaeu.ac.ae
} 
wind energy becomes one of the great energy among renewable energy in the future. One of the efficient ways to improve the performance of the wind turbine is to reduce the weight of the blades. Therefore, wind turbine blades are mainly based on fiber-reinforced polymer (FRP) composite for lightweight. Several factors expose wind turbine blades to the fatigue phenomena, which can be summarized as shown below [5]: 1. Long and flexible structures; 2. Vibrations in its resonant mode; 3 . Randomness in the load spectra due to the nature of the wind; 4. Continuous operation under different conditions; 5 . Low maintenance during the lifetime. Polymer matrix composites have been extensively used in the construction of large-scale wind turbine blades due to the low weight and high stiffness requirements [6]. Composite blade and its supporting spars are usually manufactured in parts and then bonded together with adhesives. Adhesively bonded wind turbine blades are subjected to static and fatigue loads under various environmental conditions. Therefore there is a need for rigorous analysis of the stress states in adhesive joints for better design [7]. It was reported that many investigators did tests to study the failure mechanism of the blades [8]. It was observed that crack initiated at the flaw areas in the adhesive, which led to unexpected structural responses regarding the joint failure and its associated strength. Since wind turbine blades are large-scale structures, it is difficult to avoid flaws in the manufacturing process, such as air bubbles in the adhesive layers [9]. Detailed local characterization and analysis, such as geometric imperfections and its associated stress intensity behaviors, are lacking due to the computational difficulty for accurate predictions [10]. In comparison with other mechanically fastened joints, such as rivet and screw, adhesive joints are considered to have relatively fewer sources of stress concentrations, higher toughness, and more uniform stresses distribution through the joined area. Adhesive joint failure observed between skin and spar was observed in the investigation that carried out on the failed sections of the blade [8]. Full-scale collapse testing was done under the flap-wise loading for a large full-scale wind turbine blade, which showed that the aerodynamic skins debonding of the adhesive joints is the primary failure mechanism causing a progressive collapse of the blades [11]. A three-point bending test for asymmetric beam was conducted for studying the adhesive performance between the shear web and the spar cap of the wind turbine rotor blade through investigating thick bond-lines [12]. Ahmed et al. [13-15], studied the impact of the interlaminar cracks on the mechanical behavior of deteriorated and rehabilitated structures using FEA. Linear elastic fracture mechanics was adopted to assess the stress intensity factor (SIF) as well as the stresses along the bonding line of the composite structure. Finite element analysis is a helpful tool that has been used for decades to predicate the failure of structural elements as well as the damage in the structure of the material under high loads [16], using a standard software package like ANSYS. Jrgensen et al. [17] developed a new ap- 
proach that allows the residual stress to be determined on a single test specimen for transverse cracks in adhesive joints for wind turbine blades. Prediction and simulation of the damage initiation and evolution were investigated by using a developed finite element model [18]. Engineering approaches for adhesive joint have been of great interest. Geometrical nonlinear and interlaminar progressive failure finite element analysis of a generic wind turbine blade undergoing a static flap-wise load, which was found that showed excellent correlation with the experimental results and observations in the pre-instability response. Ji and Han [19] assessed the structural integrity of composite components of a wind turbine blade that were assembled with adhesive blades in order to investigate fracture of joints. Finite element analysis based on fracture mechanics was conducted and developed to predict damage initiation and propagation to characterize the failure of an adhesive joint for a wind turbine blade. It was reported that surface delamination mechanism was the primary cause of failure [20]. The performance of adhesive joints of the carbon/epoxy wind turbine blade subjected to combined bending and tension loadings was investigated through FEM. Jrgensen et al. [21] presented a novel approach that was used in a generic tunneling crack tool for the prediction of crack growth rates for tunneling cracks propagating across a bond-line in a wind turbine blade under high cyclic loadings. Masmanidis and Theodore [22] predicted the failure of scarf joints for wind turbine blade repair of debonding in joints using a continuum damage model for simulating propagation that was performed using stress analysis plane strain elements of FE. Using evidencebased analytical procedures has increased their capability to identify the probable causes of incidents involving engineering structures [23], which can be a reference for design implementation by offering a perspective that considers factors other than the common concerns of quality, cost, and duration of work. Therefore, they reduce the difficulties caused by risks of future damage or the follow-up management of incidents [24]. Tarfaoui et al. [25] studied the damage of fiberglass composite blades for an offshore wind turbine using finite element, where implicit has been employed to simulate the response of blades for a sound knowledge of the mechanical behavior of the structures and then localize the susceptible sections, where FEA is widely used in aeronautics, civil and architecture engineering, and energy [26].

The present work aims to use 2D Linear Elastic Fracture Mechanics to investigate a pre-cracked wind turbine blade with edge crack located at the tip of the adhesive layer to evaluate the composite structure integrity. Therefore, Stress Intensity Factors (SIF) was adopted in this analysis, since it is considered as one of the fundamental problems in Linear Elastic Fracture Mechanics (LEFM) [27], where two principal approaches are known for a SIF calculation: local, based on the use of displacements or tractions near to the crack tip; and global or energy methods, which are based on the calculation of the energy release rate in terms of a crack growing. The stress 
intensity factor (SIF) has been estimated for both modes I and II. Besides, the study evaluated the interlaminar stresses, i.e., the peel as well as the shear stress along with the adhesive layer. A comparison was made with a deteriorated wind turbine blade with an embedded semi-elliptical hole $(1 \mathrm{~mm}$ by $0.5 \mathrm{~mm})$ positioned at $1.25 \mathrm{~mm}$ away from the adhesive tip. It has been revealed that the adopted method showed reliable results in comparison with the 3D analysis that would minimize efforts and cost, especially in the early stages of the design process.

\section{Linear Elastic Fracture Mechanics}

If the composite system is assumed to be a linear elastic material, the linear elastic fracture mechanics approach can be applied, and the SIF parameter can be used as a characterizing parameter for the flawed material. Linear elastic fracture mechanics technology is based on an analytical procedure that relates the stress field magnitude and distribution in the vicinity of a crack tip to the nominal stress applied to the structural member, size, shape, and orientation of the crack and material properties. The fracture is usually studied in terms of the strain energy release rate by using the methods of linear-elastic fracture mechanics. The underlying assumption of linearelastic fracture mechanics is that the relation between the strains and the stresses can be expressed by Hooke's law [28]. The fundamental principle of the fracture mechanics is that the stress field ahead of a sharp crack in structural member can be represented by a single parameter, which is the stress intensity factor $\operatorname{SIF}(K)$. This parameter is a function of both the nominal stress level in the member and the size of the crack.

Fracture mechanics is generally based on two types of analysis [29], namely residual strength analysis to determine the maximum crack size that can be tolerated, and fatigue crack growth analysis to calculate the time for crack growth from a particular initial crack size until the maximum tolerable crack size in order to determine the safe life, that is based on SIF, which can always be expressed as:

$$
K_{I}=\sigma Y \sqrt{\pi s},
$$

where $Y$ is a dimensionless factor that depends upon the geometry of the body and the crack size. The problem resides in the determination of $Y$. Many structural configurations are so complicated that a solution may not be available in handbooks. Consequently, the finite element method was still a powerful tool to determine crack tip stress fields in complicated geometry and determine the SIFs.

Basically, there are two different approaches to determine SIF by the finite element method [29]. One is the direct method in which SIF is calculated from the stress field or the displacement field. The second is an indirect method in which SIF 
is derived from another parameter, the line integral (J-Integral). In this study, the direct approach is considered. The displacement method involves a correlation of the finite element nodal point displacements with the well-known crack tip displacement equation. For mode I, the crack tip displacement can be written as

$$
U_{i}=\frac{K_{I}}{2 G} \sqrt{\frac{r}{2 \pi}} f_{i}(\theta),
$$

where $U_{1}=u$ and $U_{2}=v$.

$$
\begin{aligned}
& f_{1}=\cos (\theta / 2)\left[k-1+2 \sin ^{2}(\theta / 2)\right] \text { and } \\
& f_{2}=\sin (\theta / 2)\left[k+1-2 \cos ^{2}(\theta / 2)\right] .
\end{aligned}
$$

By substituting a nodal point displacement $U_{i}$ at some point $(r, \theta)$ near the crack tip into Eq. (2) a quantity $K_{I}$ can be calculated as:

$$
K_{I}=\sqrt{\frac{2 \pi}{r}} \frac{2 G U_{i}}{f_{i}(\theta)} .
$$

From plots of $K_{I}$ as a function of $r$ for fixed values of $\theta$ and a particular displacement component, estimates of $K_{I}$ can be made. If the exact theoretical displacement is substituted in Eq. (1), then the value of $K_{I}$ obtained as $r$ approaches zero would be the precise value of $K_{I}$. Since the FE displacements are somewhat inaccurate at a negligible distance from the crack tip, this process is not useable. Instead, a tangent extrapolating of the $K_{I}$ curve is used to estimate $K_{I}$ [30]. The most accurate estimates are obtained from $K_{I}$ curve corresponding to the normal displacement on the crack surface at $\theta=180^{\circ}$ [31]. The stress method for determining the crack tip SIF involves a correlation of the finite element nodal stresses with the well-known crack tip stress equations.

For mode $I$, the crack tip stress can be expressed as

$$
\sigma_{i j}=\frac{K_{I}}{\sqrt{2 \pi r}} f_{i j}(\theta),
$$

where $\sigma_{11}=\sigma_{x x}, \sigma_{22}=\sigma_{y y}, \sigma_{12}=\sigma_{x y}$.

Nodal point stresses $\sigma_{i j}$ in the vicinity of the crack tip can be substituted into Eq. (5) and values of $K_{I}$ can be calculated from

$$
K_{I}=\frac{\sqrt{2 \pi r}}{f_{i j}(\theta)} \sigma_{i j} .
$$

Due to the inability of the FEM to represent the stress singularity conditions at the crack tip, the $K_{I}$ curve for $r$ greater than zero must again be extrapolated back 
to $r=0$. Accurate evaluation of the SIF by stress method could be obtained from plotting $K_{I}$ curve corresponding to the normal stress on the crack tip $\left(\sigma_{y}\right)$ at an angle $\theta=0^{\circ}[32]$

\section{Finite Element Modelling of Wind Turbine Blade}

FEA has been one of the most common numerical tools in the field of fracture mechanics since the early 1960s [33]. Researchers have been developing new techniques to improve the accuracy of FEM in application to fracture mechanics $[34,35]$. In the adhesive joints, the failure usually starts at edges of the adhesive layer. The reason for this is the higher peeling stress values that occur at the edge of the adhesive region. Therefore, due to the importance of the adhesively bonded joints, the $2 \mathrm{D}$ finite element method was used to investigate the effects of the bonding joints that are partly embedded in the adherent on the tensile behavior of reinforcing plate adhesive joint [36]. Basically, the blade is subjected to drag and lift forces and consists of a composite shell supported by spar through adhesive to avoid the blade's buckling, as shown in Fig. 1.

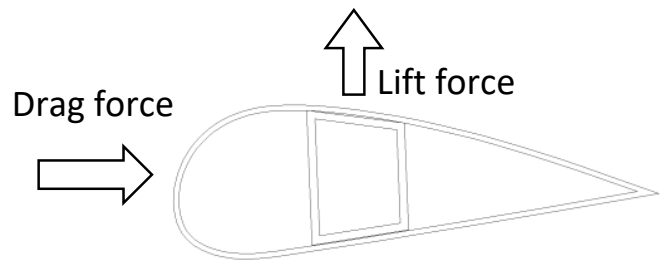

Fig. 1: Wind turbine blade profile with the applied forces.

The three-dimensional geometry investigated wind turbine blade is illustrated in Fig. 2, which shows the supporting spar bonded to the composite shell through the adhesive layer.

Figure 3 illustrates the dimensional details of the wind turbine composite structure adopted in the analysis.

Basically, when the airstrikes the blade in an angle $\alpha_{A}$ that is named the angle of attack. The reference line for the angle on the blade is most often the chord line for blade data [37]. The force on the blade $F$ can be divided into two components, the lift force $F_{L}$ and the drag force $F_{D}$ and the lift force is per definition perpendicular to the wind direction, as shown in Fig. 4.

The lift force can be calculated as [38]

$$
F_{L}=C_{L} \frac{1}{2} \rho w^{2}(b c),
$$




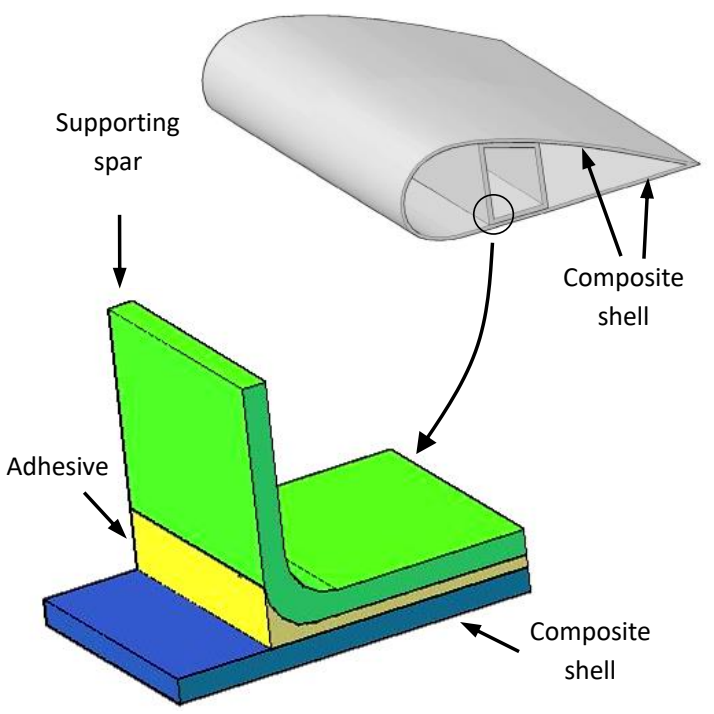

Fig. 2: 3D model of the wind turbine blade.

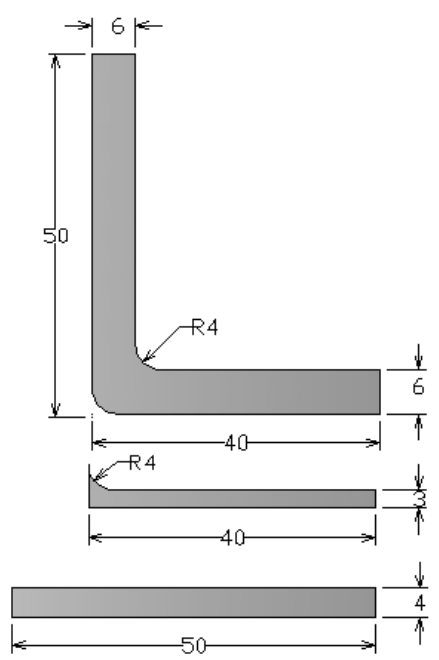

Fig. 3: Dimensions of wind turbine composite blade.

where $C_{L}$ is the "coefficient of lift", $\rho$ is the density of air, $w$ the relative wind speed, $b$ the width of the blade section, and $c$ the length of the chord line. Similar for the drag force

$$
F_{D}=C_{D} \frac{1}{2} \rho w^{2}(b c) .
$$




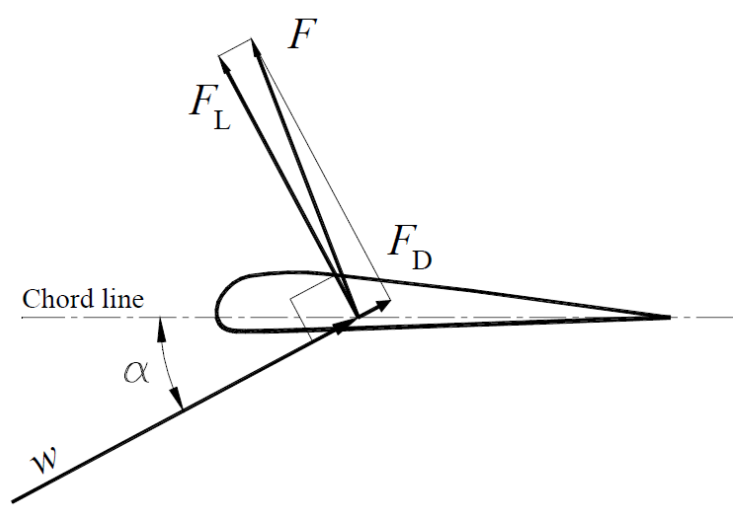

Fig. 4: The angle of attack of the blade.

The coefficient of lift and drag both depend on the angle of attack. For angles of attack higher than typically, $15-20^{\circ}$, the air is no longer attached to the blade, a phenomenon called "stall". The ratio $C_{L} / C_{D}$ is called the "glide ratio", i.e., $G R=$ $C_{L} / C_{D}$. Typically we are interested in a high glide ratio for wind turbines as well as for airplanes. Values up to 100 or higher are not uncommon, and the angles of attack giving maximum are typical in the range $5-10^{\circ}$.

For the cross-section analysis, mixed displacement-force analytical approach formulated by Jung et al. [39] was adopted for the cross-section analysis, where its advantages are that it can work with open or closed profiles, the thickness of the shell is considered, and considering the bending and torsion moments. Therefore, by considering the thin-walled assumption, the out-of-plane shear forces are assumed negligible. The laminate constitutive equation of a plate can be formulated as

$$
\left[\begin{array}{l}
N_{x x} \\
N_{s s} \\
N_{x s} \\
M_{x x} \\
M_{s s} \\
M_{x s}
\end{array}\right]=\left[\begin{array}{llllll}
A_{11} & A_{12} & A_{16} & B_{11} & A_{12} & B_{16} \\
A_{21} & A_{22} & A_{26} & B_{21} & B_{22} & B_{26} \\
A_{61} & A_{62} & A_{66} & B_{61} & B_{62} & B_{66} \\
B_{11} & B_{12} & B_{16} & D_{11} & D_{12} & D_{16} \\
B_{21} & B_{22} & B_{26} & D_{21} & D_{22} & D_{26} \\
B_{61} & B_{62} & B_{66} & D_{61} & D_{62} & D_{66}
\end{array}\right]\left[\begin{array}{c}
\epsilon_{x x} \\
\epsilon_{s s} \\
\gamma_{x s} \\
k_{x x} \\
k_{s s} \\
k_{x s}
\end{array}\right],
$$

where $N_{s s}$ and $M_{s s}$ in Eq. (9) can be considered negligible in the plate as well. However, at a specific lay-up configuration, it is pointed out that a significant error may happen in the torsional stiffness by neglecting the bending moment $M_{s s}$ [40-42]. 
Then, by reducing $N_{s s}$ and by the semi-inversion, the equation can be written as

$$
\left[\begin{array}{c}
N_{x x} \\
M_{x x} \\
M_{x s} \\
\gamma_{x s} \\
k_{s s}
\end{array}\right]=\left[\begin{array}{ccccc}
A_{n \epsilon} & A_{n k} & A_{n \emptyset} & A_{n \gamma} & A_{n \tau} \\
A_{n k} & A_{m k} & A_{m \emptyset} & A_{m \gamma} & A_{m \tau} \\
A_{n \emptyset} & A_{m \emptyset} & A_{\emptyset \emptyset} & A_{\emptyset \gamma} & A_{\emptyset \tau} \\
-A_{n \gamma} & -A_{m \gamma} & -A_{\emptyset \gamma} & A_{\gamma \gamma} & A_{\gamma \tau} \\
-A_{n \tau} & -A_{m \tau} & -A_{\emptyset \tau} & A_{\gamma \tau} & A_{\tau \tau}
\end{array}\right]\left[\begin{array}{c}
\epsilon_{x x} \\
k_{x x} \\
k_{x s} \\
N_{x s} \\
M_{s s}
\end{array}\right] .
$$

The right-hand side column vector of Eq. (10) can be formulated in the shape of beam strains as following:

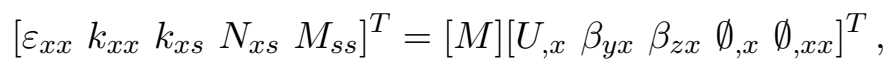

where the transfer matrix $[M]$ can be derived from the geometric relationships and continuity [39]. The beam strain terms, $U, \beta \mathbf{y}, \beta \mathrm{z}$, and $\emptyset$, are axial displacement, rotation about $y$, rotation about $z$, and twist, respectively. The subscripts $x$ in beam strains indicate the partial differential operator. Subsequently, by the virtual work principle with integration along the contour of the cross-section, the section stiffness matrix can be derived as Eq. (11) [39]

$$
\left[\begin{array}{c}
N \\
M_{y} \\
M_{z} \\
T \\
M_{\omega}
\end{array}\right]=\left[\begin{array}{lllll}
k_{11} & k_{12} & k_{13} & k_{14} & k_{15} \\
& k_{22} & k_{23} & k_{24} & k_{25} \\
& & k_{33} & k_{34} & k_{35} \\
& & & k_{44} & k_{45} \\
\text { sym. } & & & & k_{55}
\end{array}\right]\left[\begin{array}{c}
U_{, x} \\
\beta_{y, x} \\
\beta_{z, x} \\
\emptyset_{, x} \\
\emptyset_{, x x}
\end{array}\right] .
$$

The section stiffness matrix contains five external forces and the corresponding deformations, where $N$ is the tensile force along $\vec{x} ; M_{y}$ and $M_{z}$ are the bending moments about $\vec{y}$ and $\vec{z}$, respectively; $T$ and $M_{\omega}$ are the St. Venant twisting moment and Vlasov bimoment [43]. By using the analytical method, it is convenient to determine the stiffness of corresponding deformations in diagonal terms as well as the coupling intensity in off-diagonal terms.

The structural bonded joint that has been modeled is shown schematically in Fig. 5.

The assumption adopted for the structural modeling for the adherents is that beams or plates in cylindrical bending, which are described by the use of ordinary 'Kirchhoff'; plate theory where is considered as generally orthotropic laminates using classical lamination theory. Besides, the laminates are assumed to obey linear elastic constitutive laws, and that the strains are small, and the rotations are minimal. On the other hand, for the adhesive layer, it is modeled as continuously distributed linear tension and shear springs inclusion linear adhesive properties with a von Mises 


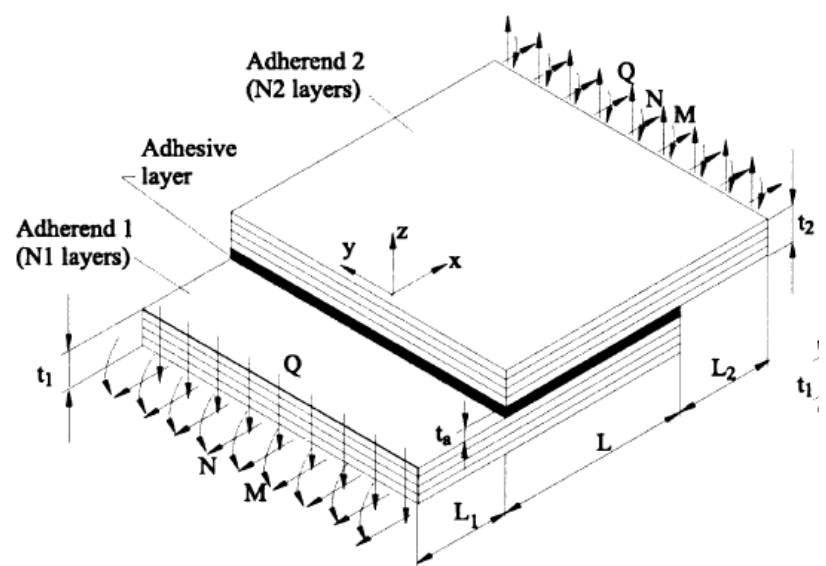

Fig. 5: Representation of the different bonded joints analyzed.

yield criterion. The system of governing equations is set up for the adherents that are modeled as plates. In general, for modeling of adherents as plates in cylindrical, as shown in Fig. 6, cylindrical bending can be defined as a wide plate (in the $y$-direction), where the displacement field can be described as a function of the longitudinal coordinate only.

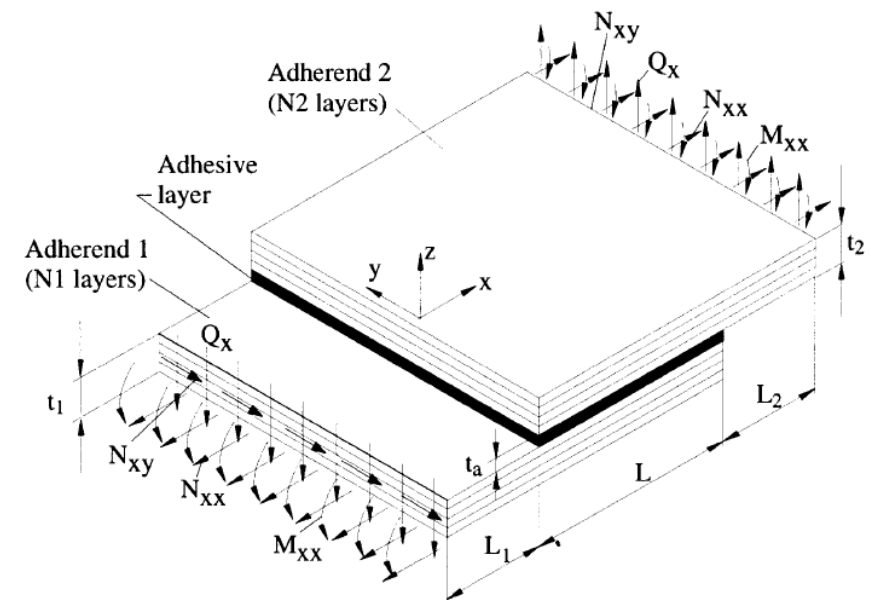

Fig. 6: Bonded single lap joint subjected to general loading conditions.

The displacement field in the width directions will be uniform, as a consequence. Therefore, the displacement field can be defined as

$$
u_{0}^{i}=u_{0}^{i}(x), \quad v_{0}^{i}=v_{0}^{i}(x), \quad w^{i}=w^{i}(x),
$$


where $u_{0}$ is the midplane displacement in the longitudinal direction ( $x$-direction), $v_{0}$ is the midplane displacement in the width direction ( $y$-direction), and $w$ is the displacement in the transverse direction ( $z$-direction). The displacement components $u_{0}, v_{0}, w$ are all defined relative to the middle surfaces of the laminates, and $i$ corresponds to the laminate/adherend number.

As a consequence of this, the following holds:

$$
u_{0, y}^{i}=v_{0, y}^{i}=w_{, y}^{i}=w_{, y y}^{i}=0 .
$$

The boundary conditions at the boundaries in the width direction are not well defined within the concept of bending. However, it is assumed that there are some restrictive constraints on the boundaries, such that they are not capable of moving and rotating freely. It should be noted that the concept of cylindrical bending; is not unique and that other definitions than the one used in the present formulation can be adopted [44]. Substitution of the quantities in Eq. (13) into the constitutive relations for a laminated composite material [44] gives the constitutive relations for a laminate $(i)$ in bending

$$
\begin{aligned}
& N_{x x}^{i}=A_{11}^{i} u_{0, x}^{i}+A_{16}^{i} v_{0, x}^{i}-B_{11}^{i} w_{, x x}^{i} \\
& N_{y y}^{i}=A_{12}^{i} u_{0, x}^{i}+A_{26}^{i} v_{0, x}^{i}-B_{12}^{i} w_{, x x}^{i} \\
& N_{x y}^{i}=A_{16}^{i} u_{0, x}^{i}+A_{66}^{i} v_{0, x}^{i}-B_{16}^{i} w_{, x x}^{i} \\
& M_{x x}^{i}=B_{11}^{i} u_{0, x}^{i}+B_{16}^{i} v_{0, x}^{i}-D_{12}^{i} w_{, x x}^{i} \\
& M_{y y}^{i}=B_{12}^{i} u_{0, x}^{i}+B_{26}^{i} v_{0, x}^{i}-D_{12}^{i} w_{, x x}^{i} \\
& M_{x y}^{i}=B_{16}^{i} u_{0, x}^{i}+B_{66}^{i} v_{0, x}^{i}-D_{11}^{i} w_{, x x}^{i}
\end{aligned}
$$

where $A_{j k}^{i}, B_{j k}^{i}$, and $D_{j k}^{i}(j, k=1,2,6)$ are the extensional, coupling, and the flexural rigidities as defined by classical lamination theory, as described by Jones [45]. $N_{x x}^{i}, N_{y y}^{i}$, and $N_{x y}^{i}$ are the in-plane stress resultants, and $M_{x x}^{i}, M_{y y}^{i}$, and $M_{x y}^{i}$ are the moment resultants. For the advanced joint types such as a scarfed or stepped lap, the rigidities $A_{j k}^{i}, B_{j k}^{i}$ and $D_{j k}^{i}(j, k=1,2,6)$ are determined as functions of the longitudinal direction of the joint within the overlap zone, since the adherend thicknesses are variable within the overlap. From the Kirchhoff assumptions, the following kinematic relations for the laminates are derived

$$
u^{i}=u_{0}^{i}+z \beta_{x,}^{i}, \quad \beta_{x}^{i}=-w_{, x}^{i}, \quad \beta_{y}^{i}=0,
$$

where $u^{i}$ is the longitudinal displacement, $u_{0}^{i}$ is the longitudinal displacement of the midplane, and $w^{i}$ is the vertical displacement of the $i_{t h}$ laminate.

Basically, linear elastic behavior is used to model the properties of the adhesive layer in the joint analysis. Besides, the supporting spar and the composite shell are 
Table 1: Materials properties for the composite blade and the adhesive [33].

\begin{tabular}{lcc}
\hline \hline Properties & Composite shell & Adhesive \\
\hline Longitudinal modulus $E_{1}$ & $145 \mathrm{GPa}$ & $1.1 \mathrm{GPa}$ \\
Transverse modulus $E_{2}$ & $10 \mathrm{GPa}$ & - \\
Transverse modulus $E_{3}$ & $10 \mathrm{GPa}$ & - \\
Shear modulus $G_{12}$ & $7 \mathrm{GPa}$ & 0.382 \\
Shear modulus $G_{13}$ & $7 \mathrm{GPa}$ & - \\
Shear modulus $G_{23}$ & $3.7 \mathrm{GPa}$ & - \\
Poisson's ratio $\nu_{12}$ & 0.25 & 0.44 \\
Poisson's ratio $\nu_{13}$ & 0.25 & - \\
Poisson's ratio $\nu_{23}$ & 0.5 & - \\
\hline \hline
\end{tabular}

considered to have orthotropic characteristics through the analysis, whereas for the adhesive layer is taken as isotropic. Table 1 shows the properties adopted.

Two dimensional, plane strain finite element model was adopted in the analysis [46] using 8-node quadrilateral (Solid 183) instead of the 3D model. In general, the essential element size chosen was $1 \mathrm{~mm}$ for the supporting spar and the composite shell as well, whereas for the adhesive layer was $0.5 \mathrm{~mm}$ except at the crack tip, where the mesh was done according to the software macro. This is depicted in Fig. 7.

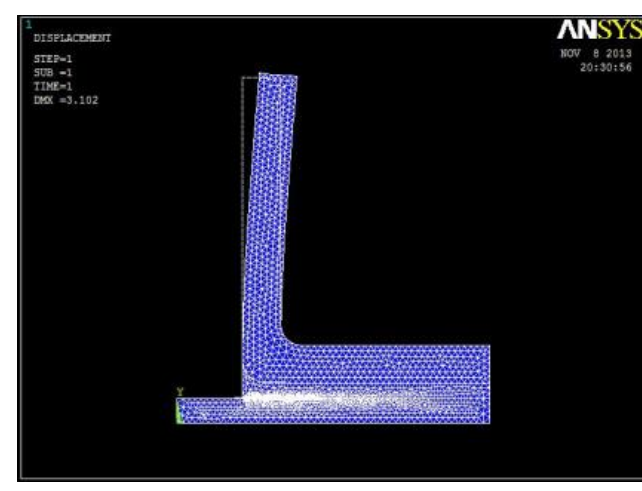

(a)

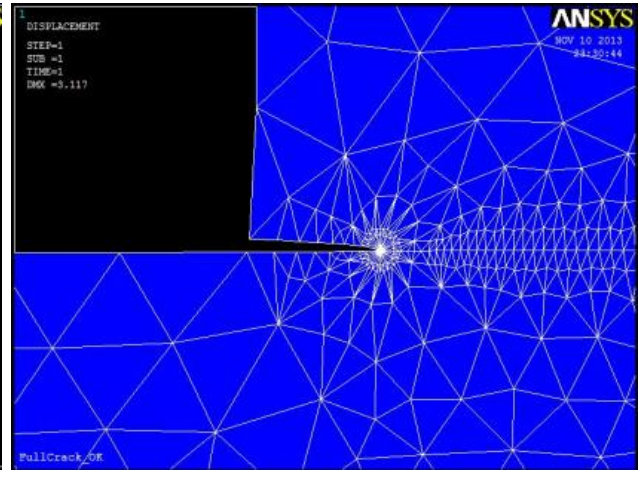

(b)

Fig. 7: a) FE model of the blade, b) FE mesh at the crack tip.

The applied combined loads on the proposed structure were tensile stress of $100 \mathrm{MPa}$ along the $y$-axis of the supporting spar, which simulates the lifting and the drag force on the wind turbine blade resulted from the wind-induced pressure difference [47]. Besides, a $3 \mathrm{~mm}$ displacement along the $x$-axis was applied at the end of the sup- 
porting spar as well. The composite shell was constrained in all directions. Taking into account that the condition of the bonding lines is considered to have a perfect adhesion between the adhesive and the composite shell one time and the adhesive and the supporting spar in the second time. In this context, it is important to clarify that instead of analyzing the influence of through-thickness elliptical void located at the adhesive layer on the interlaminar stresses and the SIF, an edge crack was studied, which was located between the adhesive layer and the composite shell, as shown in Fig. 8.

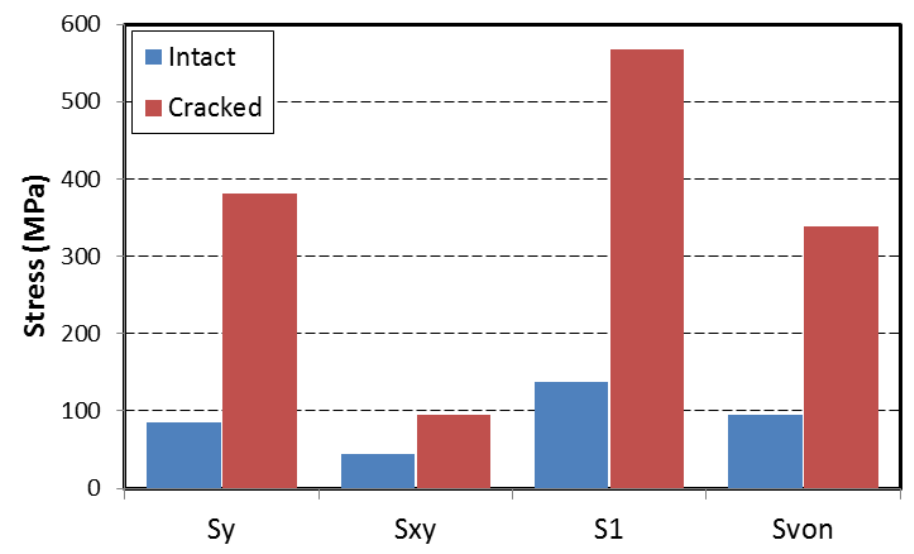

Fig. 8: Fractured blade with edge crack at the adhesive tip.

Knowing that the crack length was analogs to studied case [9], except it starts from the adhesive tip. Linear elastic fracture mechanics (LEFM) was adopted to estimate SIF at the crack tip for mode I and II. Besides, the influence of the adhesive layer stiffness, i.e., shear modules, were explored. Stresses, shear, peel, principal as well as Von Misses were estimated at the adhesive tip for the intact case, whereas evaluated at the crack tip for the fractured example.

\section{RESUlts AND Discussion}

The first stage of the analysis was to investigate an intact model, i.e., without any void or crack, in order to locate the maximum stresses in the model, especially the critical points close to the adhesive layers. It has been seen that the maximum stresses exist at the adhesive tip because of the lack of fillet in the studied model, as shown in Fig. 9.

It is depicted that the high values of the peeling stress in comparison with the applied one, whereas the 1st principal stresses already exceed the applied stresses. Von Misses stresses approaches the applied stresses. Shear stresses more than $40 \%$ 


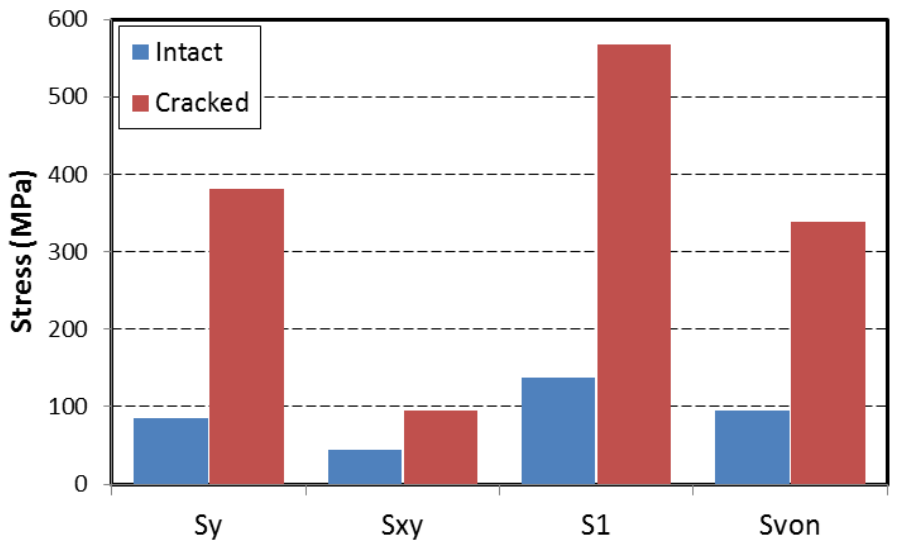

Fig. 9: Stresses at the adhesive tip for intact, and the crack tip for the fractured wind turbine blade.

of the applied stress, and this is clear evidence that this point is critical in the geometry where there is a big opportunity for crack initiation and prorogation. On the other hand, by introducing an edge crack at the adhesive tip, between the adhesive layer and the composite shell, a consequence of the fact that a considerable increase in the stresses at the crack tip was observed as illustrated in Fig. 6, which illustrates maximum stresses. It can be interpreted in comparison with the intact case, that an increase of 348\% was observed in the peel stresses, and 90\% shown in the shear stresses, whereas $314 \%$ in the 1 st principal stresses, and eventually $278 \%$ increase in the Von Misses stresses. In order to investigate the impact of the adhesive shear modulus on the peel and the shear stresses at the crack tip, a parametric study was carried out. The results are represented by Fig. 10, where the adhesive Young's modulus (i.e., $E_{x y}$ ) varies from 1100 to $6600 \mathrm{GPa}$, whereas the adhesive shear modulus (i.e., $G_{x y}$ ) is estimated using principles of solid mechanics.

It has been evident that with the increase of the shear modulus of the adhesive, both peel and shear stresses at the crack tip increases accordingly. In fact, the rise in the peel stresses can approach $70 \%$ as the adhesive stiffness becomes about seven times the primary value (Young's modulus 1.1 GPa), though less rise in the shear stresses up to 55\%, where observed with the same adhesive stiffness increase. The last stage of the study was to investigate using linear elastic fracture mechanics principle (LEFM), the influence of the fractured blade caused by presumed edge crack located between the adhesive layer and the composite shell, on the estimated stress intensity factor (SIF). It has been known that voids play an essential role in adhesive joint failure [48]. Therefore the present study elaborates on the impact of the esti- 


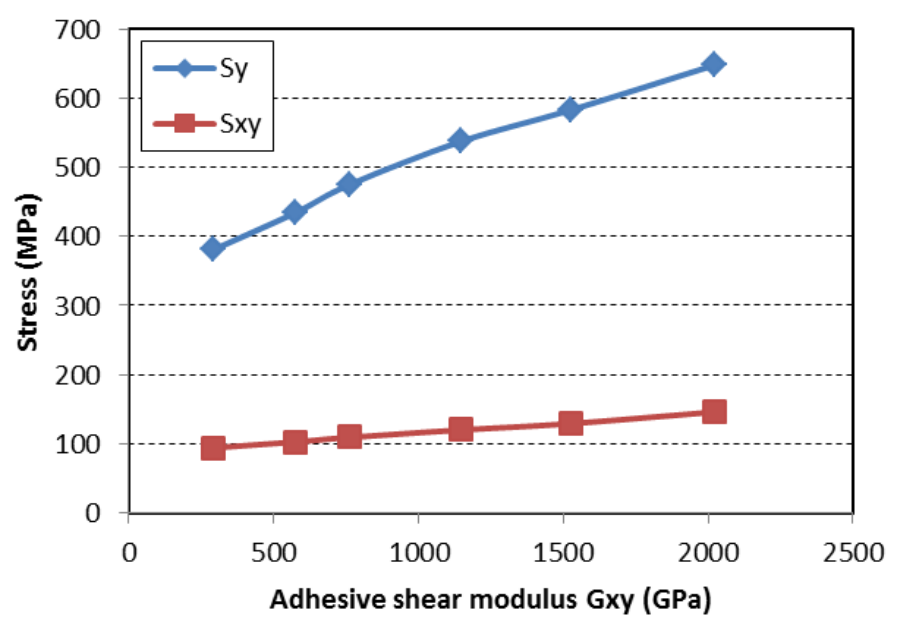

Fig. 10: Stresses at the adhesive tip for the intact blade.

mated SIF as a result of the fractured adhesive layer. Fig. 11 illustrates the location of the elliptical void $(1 \mathrm{~mm}$ by $0.5 \mathrm{~mm}$ ) positioned at $1.25 \mathrm{~mm}$ away from the adhesive tip, which was studied by Hua et al., where SIF was estimated at the main side tips of the void (i.e., Tip-1 and Tip-2).

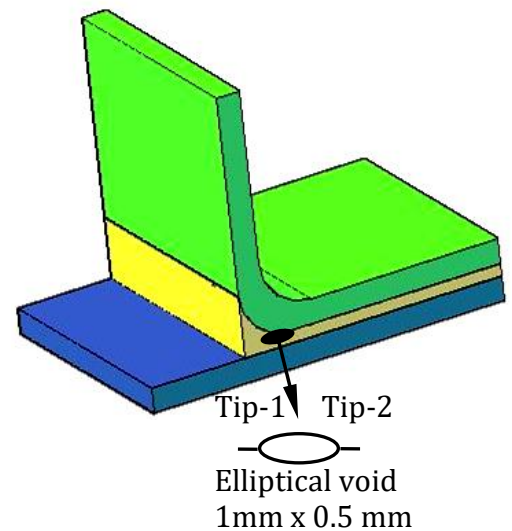

Fig. 11: Elliptical void located $1.25 \mathrm{~mm}$ from the adhesive tip [14].

Mainly due to the combined loading that the blade is subjected, it is expected to have both opening and shear mode of stress intensity factor. Therefore these two types were analyzed and compared. Clear evidence can be observed from the presence of the edge crack in increasing SIF in levels more than that caused by the void, and this can be elucidated in Fig. 12, where it is apparent that SIF of the cracked 


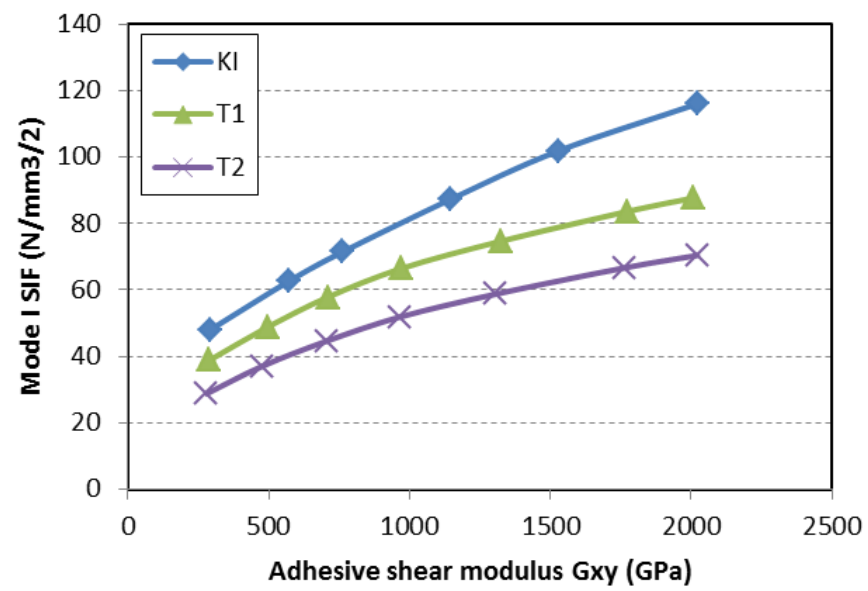

Fig. 12: Mode I SIF (KI) for fractured and voided (Tip-1(T1) and Tip-2 (T2) Ref. [13]), as a function of adhesive shear modulus.

blade overtakes and dominates the voids results.

The increase in the SIF of the fractured blade can be as $23 \%$ more than the maximum SIF for the blade with the void, i.e., at tip 1, when the adhesive stiffness was 1.1 GPa (for isotropic material, shear modulus can be estimated from Young's modulus by $G=E / 2(1+\nu)$. In contrast, it is shown to be $32 \%$ more than the maximum SIF of the voided blade at a stiffness of $5.8 \mathrm{GPa}$. Regarding the impact of the edge

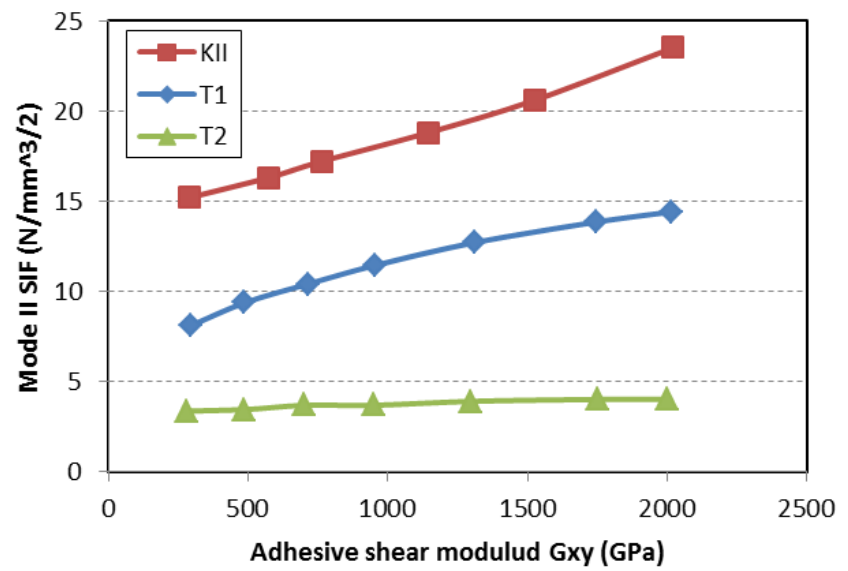

Fig. 13: Mode II SIF (KII) for fractured and with voided (tip1 and tip2 [14]) blade, as a function of adhesive shear modulus. 
crack on the mode II SIF, by the same FE model, KII was estimated at the same time of evaluating KI, and besides was analyzed in comparison with adhesive stiffness, i.e., shear modulus. Fig. 13 shows that mode II SIF, i.e., KII, exceeds the level of the SIF estimated at the ends of the trough thickness voids. Although the level of the SIF of mode II is lower that mode I, but since it is located in the weak layer, i.e., the adhesive layer, it makes the failure situation of the blade more crucial. However, this increase of mode II SIF can be seen to be 4.5 times the maximum SIF of the voided blade at the lowest value of the adhesive shear modulus and to be 5.9 times at the maximum shear modulus value.

\section{CONCLUSIONS}

The present analysis is based on using Linear Elastic Fracture Mechanics and Finite Element Analysis to evaluate the Stress Intensity Factor of an edge crack located at the tip of the adhesive layer of the wind turbine blade. The study discusses the results in comparison with a wind turbine with an embedded void. The following points summarize the investigation outcomes:

1. For the intact blade, the stresses at the adhesive tip are relatively higher than the applied, especially the peel and the principal stresses, and this would cause the initiation of the crack, especially with lack of the adhesive fillet.

2. The presence of the edge crack at the adhesive tip increases the stresses at the crack tip times than the applied stress, and this might cause the propagation of the crack.

3. One of the main reasons for the failure of the constructions made of composite materials is the initiation and propagation of cracks. In order to investigate these cracks, fracture mechanics methods have been used [31]. It has been demonstrated in this investigation that Mode I and II SIF of the fractured blade show always higher levels than the corresponding SIF of the blade with avoid.

Cracks may significantly reduce the strength and stiffness of the structural element, especially composite since nowadays, most of the wind turbine components are made from the composite section and material [49]. Thus, the load-bearing capacity of a structure is highly dependent on the fracture toughness properties of the structural component. This would cause a problem that leads to failure in case of any cracks or tiny holes that may exist in the bonded joints.

Eventually, it has been revealed by the study that two-dimensional analysis can be implemented effectively to analysis deteriorated wind turbine blades caused by embedded gravities within the adhesive layer of the bonded composite structure, where 
the results illustrated critical levels of the SIF associated with the cracked bonded components that could be used later on to estimate fatigue lifetime easily rather than adopting three-dimensional analysis.

\section{ACKNOWLEDGMENT}

The author would like to thank Dr. Linxia Gu, Department of Mechanical and Materials Engineering, University of Nebraska-Lincoln, Lincoln, for the support over the stages of the study.

\section{REFERENCES}

[1] A.R. JHA (2011) "Wind turbine technology". Boca Raton, CRC, London, Taylor \& Francis.

[2] W.K. Ahmed (2013) Mechanical modelling of wind turbine: Comparative study. International Journal of Renewable Energy Research 3 94-97.

[3] M.M. SHOKRIEH, R. RAFIEE (2006) Simulation of fatigue failure in a full composite wind turbine blade, Composite Structures 74 332-342.

[4] Y.M. JI, K.S. HAN (2014) Fracture mechanics approach for failure of adhesive joints in wind turbine blades. Energy Renewable Energy 65 23-28.

[5] D.A. SPERA (2009) "Wind turbine technology: fundamental concepts of wind turbine engineering". New York, ASME Press.

[6] K.L. EDWARDS (1998) A brief insight into the selection and use of engineering adhesives for preliminary joint design. Materials in Engineering 19121.

[7] H.S. Da Costa Mattos, A.H. Monteiro, R. Palazzetti (2012) Failure analysis of adhesively bonded joints in composite materials. Materials \& Design 33 242-247.

[8] L.C.T. OvergaArd, E. Lund (2010) Structural collapse of a wind turbine blade. Part B: Progressive interlaminar failure models. Composites Part A: Applied Science and Manufacturing Composites 41 271-283.

[9] Y. Hua, A.R.M. Kasavajhala, L. Gu (2013) Elastic-plastic analysis and strength evaluation of adhesive joints in wind turbine blades. Composites Part B: Engineering 44 650-656.

[10] D. Ouinas, B. Bachir Bouiadjra, S. Himouri, N. Benderdouche (2012) Progressive edge cracked aluminium plate repaired with adhesively bonded composite patch under full width disbond. Composites, Part B: Engineering 43 805-811.

[11] J. YANG (2013) Structural investigation of composite wind turbine blade considering structural collapse in full-scale static tests. Composite Structures 97 15-29.

[12] F. SAYER, A. ANTONIOU, A. VAN Wingerde (2012) Investigation of structural bond lines in wind turbine blades by sub-component tests. International Journal of Adhesion and Adhesives 37 129-135.

[13] W.K. Ahmed, A.H.I. Mourad (2015) Fracture Assessment of Strengthened Cracked Metallic Components Using FRP Stiffeners. Mechanics of Composite Materials 51 301312. 
[14] W.K. Ahmed, W.N. Al-Rifaie, C. Rudd (2012) Structural Assessment via FEA of Bonded Steel Structures using Fiber Reinforced Epoxy Adhesive. International Journal of Material and Mechanical Engineering 4(1) 61-65.

[15] W.K. Ahmed, A.H.I. Mourad (2012) Using Fiber Reinforced Polymer to Restore Deteriorated Structural Members. International Journal of Material and Mechanical Engineering 1(1) 1-7.

[16] A.K. Lyubimov, D.A. Kozhanov (2016) Modeling the Structural Element of Flexible Woven Composites under Static Tension Using the Method of Finite Element in ANSYS. Computer Research and Modeling 8(1) 113-120.

[17] D.S. Zarouchas, A.A. Makris, D. Van Hemelrijck, F. Sayer, A.M. Van WINGERDE (2012) Investigations on the mechanical behavior of a wind rotor blade subcomponent. Composites Part B: Engineering 43 647-654.

[18] B.F. SRensen, S. Goutianos, T.K. JacobSen (2009) Strength scaling of adhesive joints in polymer-matrix composites. International Journal of Solids and Structures 46 741-761.

[19] Y.M. Ji, K.S. HAN (2014) Fracture mechanics approach for failure of adhesive joints in wind turbine blades. Renew. Energy Renewable Energy 65 23-28.

[20] J.-S. Chou, C.-K. ChiU, I.K. HuAng, K.-N. Chi (2013) Failure analysis of wind turbine blade under critical wind loads. Engineering Failure Analysis 27 99-118.

[21] J.B. Jrgensen, B.F. Srensen, C. KildegaArd (2018) Tunneling cracks in full scale wind turbine blade joints. Engineering Fracture Mechanics 189 361-376.

[22] I.T. MASMANIDIS, T.P. PHILIPPIDIS (2018) Modeling the progressive failure of scarf joints for wind turbine blade repair. Journal of Composite Materials, 62(16).

[23] N. Athiniotis, D. Lombardo, G. Clark (2010) Scientific analysis methods applied to an investigation of an aircraft accident. Engineering Failure Analysis $\mathbf{4 8} 83$.

[24] S. BROWN (2007) Forensic engineering: Reduction of risk and improving technology (for all things great \& small). Engineering Failure Analysis 14 1019-1037.

[25] M. Tarfaoui, M. Nachtane, H. Boudounit (2019) Finite Element Analysis of Composite Offshore Wind Turbine Blades Under Operating Conditions. Journal of Thermal Science and Engineering Applications 12011001.

[26] S. SANKAR, M. NATARAJ, V.P. RAJA (2011) Failure analysis of shear pins in wind turbine generator. EFA Engineering Failure Analysis 18 325-339.

[27] S. PARVANOVA (2012) Calculation of Stress Intensity Factors Based on ForceDisplacement Curve using Element Free Galerkin Method. Journal of Theoretical and Applied Mechanics 42(1) 23-40.

[28] V. Rizov (2016) Non-Linear Analysis of Mode II Fracture in the end Notched Flexure Beam. Journal of Theoretical and Applied Mechanics 46(1) 53-64.

[29] D. BROEK (2013) "Elementary engineering fracture mechanics". Springer.

[30] S.K. Chan, I.S. TubA, W.K. Wilson (1970) On the finite element method in linear fracture mechanics. Engineering Fracture Mechanics 2 1-17. 
[31] A.S. Mladensky, V.I. Risov (2012) Analysis of Mixed Mode II/III Crack in Bilayered Composite Beam. Journal of Theoretical and Applied Mechanics 42(4) 41-52.

[32] C.F. SHIH, H.G. DE LorenzI, M.D. GeRMAn (1976) Crack extension modeling with singular quadratic isoparametric elements. International Journal of Fracture 12 647651.

[33] A.R. Gu, L.M. Kasavajhala, S. Zhao (2011) Finite element analysis of cracks in aging aircraft structures with bonded composite-patch repairs. Composites, Part B $\mathbf{4 2}$ 505-510.

[34] M. Souiyah, A. Muchtar, A. Alshoaibi, A.K. Ariffin (2009) Finite element analysis of the crack propagation for solid materials. American Journal of Applied Sciences 6(7) 1396-402.

[35] K. Aslantas, S. Tasgetiren, E. Ergun, M. Topcu (2006) Computation of the stress intensity factors and life prediction for repaired cracks. Technology Research $\mathbf{1}$ $1-10$.

[36] I.S.C. BahatTin (2015) Stress Analysis of Lap Joints With Embedded Cover Plate. Journal of Theoretical and Applied Mechanics 45 91-100.

[37] D. Rommel, D. Di Maio, T, Tinga (2020). Calculating wind turbine component loads for improved life prediction. Renewable Energy 146 223-241.

[38] M.O.L. Hansen (2015) "Aerodynamics of wind turbines". London: Routledge, Taylor $\&$ Francis Group.

[39] S.N. Jung, V.T. NAgaraJ, I. Chopra (2002). Refined Structural Model for Thinand Thick-walled Composite Rotor Blades, AIAA Journal 40(1) 105-116.

[40] S.N. Jung, I.J. PARK, E.S. ShIN (2007) Theory of Thin-walled Composite Beams with Single and Double-cell Sections. Composites, Part B. Engineering 38(2) 182-192.

[41] V.V. Volovoi, D.H. Hodges (2002) Single- and Multicelled Composite Thin-walled Beams. AIAA Journal 40(5) 960-965.

[42] S.N. Jung, J.Y. LeE (2003) Closed-form Analysis of Thin-walled Composite I-Beams Considering Non-classical Effects. Composite Structures 60(1) 9-17.

[43] H. LIN, W. LAI, Y. Kuo (2010) Combined analytical and finite element beam model for wind turbine blades. Journal of Reinforced Plastics and Composites 29(16) 24222437.

[44] J.M. WhitNEY (1987) "Structural analysis of laminated anisotropic plates". Lancaster: Technomic Publishing Company.

[45] O.T. ThOMSEn (1992) Elasto-static and elasto-plastic stress analysis of adhesive bonded tubular lap joints. Composite Structures 21 249-59.

[46] S. MoAveni (2008) "Finite element analysis: theory and application with ANSYS". Hoboken, NJ: Pearson, Inc.

[47] B.F. SRENSEN, T.K. JACOBSEN (2006) "Joining structural parts of composite materials for large rotorblades". Ris $\varnothing$ National Laboratory. 
[48] A. Chadegani, R.C. Batra (2011) Analysis of adhesive-bonded single-lap joint with an interfacial crack and a void. International Journal of Adhesion and Adhesives 31 455-465.

[49] V. Rizov (2012) Fracture in Composites - An Overview (Part I). Journal of Theoretical and Applied Mechanics 42(2) 3-42. 